Special Reviews

Juntendo Medical Journal

2019. 65 (1), 12-19

\title{
Corticosteroid and Retinoid Metabolism Signalling Pathways During Decidualisation of Human Endometrial Stromal Cells
}

\author{
KEIJI KURODA ${ }^{* 1) 2}$ \\ *1) Center for Reproductive Medicine and Implantation Research, Sugiyama Clinic Shinjuku, Tokyo, Japan, \\ *2) Department of Obstetrics and Gynaecology, Juntendo University Faculty of Medicine, Tokyo, Japan
}

\begin{abstract}
In humans, the incidence of embryo wastage and pregnancy loss is extraordinarily high; therefore, pregnancy loss commonly occurs in reproductive women. Furthermore, some women suffer from recurrent pregnancy loss (RPL). In over $50 \%$ of RPL cases, no specific cause is identified even after detailed examinations. Impaired decidualisation of uterine endometrium with aberrantly high uterine natural killer (uNK) cell density is associated with unexplained RPL. Glucocorticoid includes active cortisol and inert cortisone, which are mutually catalysed by 11ß-hydroxysteroid dehydrogenase (11ßHSD). 11ßHSD isoform type 1 activates cortisone to cortisol, which binds glucocorticoid receptor (GR) and mineralocorticoid receptor (MR). During decidual transformation in human endometrial stromal cells (HESCs), 11BHSD1, but not type 2, is particularly induced by progesterone, leading to the local production of active cortisol and regulation of GR and MR gene networks. 11ßHSD1/GR signalling is a significant pathway for the regulation of uNK cells and inflammatory reaction upon decidualisation of HESCs, whereas the MR gene network includes retinoid metabolism. Retinol (vitamin A), which is hydrolysed from retinyl ester, is transformed to retinaldehyde (Rald) and generates retinoic acid (RA) following two-step oxidation. RA binds to CRABP2 and activates retinoic acid receptor (RAR), leading to cell apoptosis and senescence, whereas RA-dependent activation of PPAR $\beta / \delta$ is mediated through FABP5, leading to cell differentiation. The decidualisation of HESCs decreases the expression of CRABP2 and FABP5 and down- and upregulates RAR and PPAR $\beta / \delta$, respectively. In addition, decidualisation is associated with the upregulation of retinoid metabolism-related genes, including retinol-binding protein 4 (RBP4), cytochrome P450 26A1 (CYP26A1), RDH12 and DHRS3, suggesting that decidualisation suppresses RA signalling by decreasing key cytoplasmic-binding proteins and upregulating retinoid metabolism. Taken together, corticosteroid signalling via 11ßHSD1 induction regulates GR-specific gene networks including inflammation and angiogenesis at the implantation site. Furthermore, retinoid metabolism signalling is controlled via 11ßHSD1/MR signalling, leading to an optimal balance of cell differentiation and apoptosis, such as decidualisation of HESCs. Recently, for the treatment of unexplained RPL, the GR and MR gene networks are targeted via corticosteroid signalling, leading to modulation of uNK cells as well as retinoid metabolism signalling.
\end{abstract}

Key words: endometrium, decidualisation, 11ß-hydroxysteroid dehydrogenase type 1 (11ßHSD1), retinoid, recurrent pregnancy loss (RPL)

Funding: K.K. was supported by JSPS KAKENHI Grant Number 18K09273.

Abbreviations: 8-bromo-cAMP, 8-bromoadenosinecAMP; 11ßHSD, 11ß-hydroxysteroid dehydrogen- ase; GR, Glucocorticoid receptor; HESC, human endometrial stromal cell; IGFBP1, insulin-like growth factor-binding protein-1; MR, Mineralocorticoid receptor; P4, progesterone; PR, progesterone

Keiji Kuroda

Center for Reproductive Medicine and Implantation Research, Sugiyama Clinic Shinjuku

1-19-6 Nishishinjuku, Shinjuku-ku, Tokyo 160-0023, Japan

TEL: +81-3-5381-3000 FAX: +81-3-5381-4124 E-mail: kuroda@sugiyama.or.jp

345th Triannual Meeting of the Juntendo Medical Society: Medical Research Update〔Held on May 19, 2018〕

〔Received Aug. 2, 2018〕〔Accepted Aug. 6, 2018〕

Copyright (C) 2019 The Juntendo Medical Society. This is an open access article distributed under the terms of Creative Commons Attribution License (CC BY), which permits unrestricted use, distribution, and reproduction in any medium, provided the original source is properly credited. doi: 10.14789/jmj.2019.65.JMJ18-R13 
receptor; PRL, prolactin; RPL, recurrent pregnancy loss; uNK, uterine natural killer.

\section{Introduction}

Successful pregnancy is initiated with a mutual crosstalk between endometrial receptivity and embryo competency. In humans, the incidence of pre-implantation embryo wastage, pre-clinical pregnancy loss, and miscarriage is extraordinarily high. The incidence rate of embryo wastage is estimated to be $30 \%$ before implantation (implantation failure), $30 \%$ until 6 weeks of pregnancy (biochemical pregnancy loss), and 10\%-15\% primarily before 12 weeks of gestation (clinical pregnancy loss $)^{1)}$ (Figure-1). Chromosomal abnormalities in human embryos account for the high incidence of early embryo and pregnancy loss ${ }^{2)}$ ). Of women who have experienced pregnancy, 38\% have a history of spontaneous abortion; therefore, pregnancy loss is common in reproductive women ${ }^{4)}$. Furthermore, some women suffer from repeated pregnancy losses. The European Society for Human Reproduction and Embryology
(ESHRE) defines recurrent pregnancy loss (RPL) as $\geq 3$ pregnancy losses ${ }^{5)}$. The incidence rates of two and three consecutive accidental pregnancy losses is estimated to be $4.2 \%$ and $0.9 \%$, respectively ${ }^{4)}$. Ogasawara $e t$ al. reported that an increase in the number of pregnancy losses resulted in the decrease in the likelihood of abnormal embryonic karyotypes and an increase in the frequency of subsequent pregnancy loss ${ }^{6}$. It is highly probable that couples with a history of multiple pregnancy losses have parental, not foetal, risk factors for pregnancy loss ${ }^{7)}$. A Japanese RPL research group at the Health, Labour and Welfare Ministry of Japan investigated the frequency of risk factors for RPL and demonstrated rates of approximately $25 \%$ for thrombophilia, including antiphospholipid antibody syndrome, $7.8 \%$ for organic factors such as uterine malformation, and $6.8 \%$ for thyroid disorder (http://fuiku.jp/study/). However, in over $50 \%$ of RPL cases, no specific cause is identified even after detailed examinations ${ }^{8}$. Candidate risk factors for unexplained RPL include impaired intrauterine circumstances and impaired decidualisation of uterine endometrium ${ }^{7)}$. This

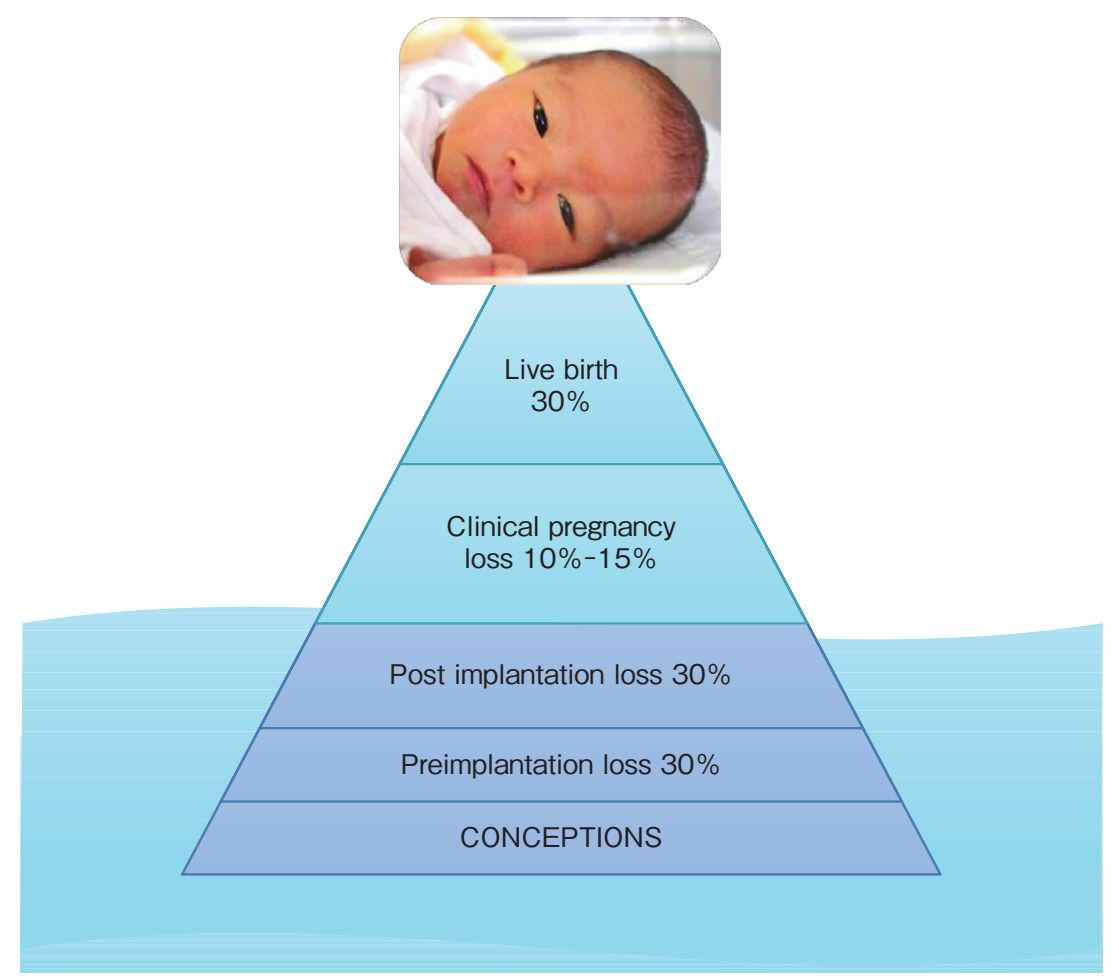

Figure-1 Embryo wastage iceberg

In humans, the incidence of embryo wastage and pregnancy loss is extraordinarily high. The incidence rate of embryo wastage is estimated to be $30 \%$ before implantation, $30 \%$ until 6 weeks of pregnancy (biochemical pregnancy loss), and 10\%-15\% primarily before 12 weeks of gestation (clinical pregnancy loss). 
review aimed to demonstrate the importance of corticosteroid and retinoid signalling pathways in the decidualisation of human endometrium for implantation and maintenance of early pregnancy.

\section{Decidualisation and recurrent reproductive failure}

Recent studies reported that decidualised human endometrial stromal cells (HESCs) serve as biosensors of embryo quality at implantation ${ }^{9)-12}$. Human developmentally inappropriate embryos may suppress the secretion of implantation regulators, including inflammatory cytokines from decidualising HESCs ${ }^{9)}$. However, the endometrium of women with a history of unexplained RPL has high migratory activity to an embryo, regardless of its quality; therefore, it disables the ability to avoid a low-quality embryo ${ }^{10)}$. In addition, several publications have reported a relationship between recurrent reproductive failure and aberrantly high uterine natural killer ( $\mathrm{uNK})$ cell $\left(\mathrm{CD} 56^{\text {bright }}\right.$ and $\mathrm{CD} 16^{\mathrm{dim}}$ ) density in mid-luteal endometrial cells ${ }^{13)-17)}$. uNK cells, an important part of the innate immune system, are the most abundant immune cells in pre-implantation endometrium and early pregnancy decidua ${ }^{18)}$. Further, uNK cells are believed to have a regulatory function in inflammation and angiogenesis via interleukin (IL) -11 and IL-15 secretion ${ }^{15)}{ }^{19)}$-21). Disordered inflammatory regulators, IL33/ST2 in IL1 superfamily are associated with specific reproductive disorders ${ }^{22)}$. From a genetic viewpoint, NLRP2 and NLRP7 genes, which regulate the production of IL1 1 , are also reportedly associated with unexplained $\mathrm{RPL}^{23)}$. Therefore, there are obvious evidences suggesting an intimate interaction between RPL and inappropriate inflammation, which leads to abnormal angiogenesis, in mid-luteal endometrial stromal cells during periimplantation and decidualisation.

\section{Corticosteroid signalling upon decidualisation of HESCs}

Glucocorticoids indicate the effects of potent anti-inflammation and immunosuppression. Endogenous glucocorticoids ensure significant functions in embryo implantation, placentation, and foetal growth during the course of early pregnancy.

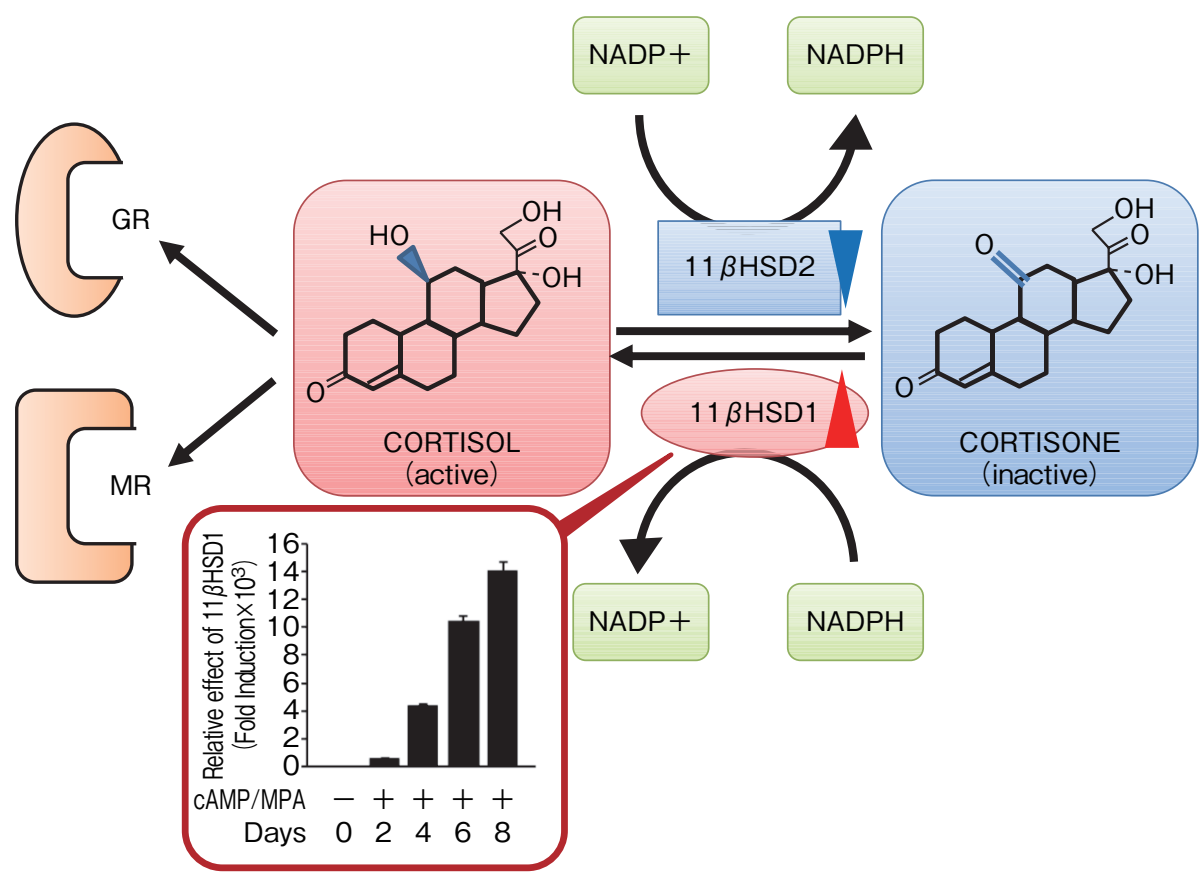

Figure-2 Cortisol-cortisone interconversion

Glucocorticoid includes active cortisol and inert cortisone, which are mutually catalysed by $11 \beta$-hydroxysteroid dehydrogenase (11BHSD). NADPH-dependent 11BHSD isoform type 1 activates cortisone to cortisol, which binds glucocorticoid receptor (GR) and mineralocorticoid receptor (MR). In contrast, 11ßHSD type 2 inactivates cortisol. During decidual transformation in endometrial stromal cells, $11 \beta \mathrm{HSD} 1$, but not type 2 , is highly induced by progesterone, leading to the local production of active cortisol. Red arrow: upregulation of the gene upon decidualisation. Blue arrow: downregulation of the gene. 
Glucocorticoid treatment reportedly prevents RPL on the basis of its ability to inhibit excess uNK cells and angiogenesis during the peri-implantation window ${ }^{14)}{ }^{24)}$. Glucocorticoid includes active cortisol and inert cortisone, which are catalysed mutually by 11ß-hydroxysteroid dehydrogenase (11ßHSD). The NADPH-dependent 11BHSD isoform type 1 activates cortisone to cortisol, which binds glucocorticoid receptor (GR); however, cortisol has a high affinity for mineralocorticoid receptor $(\mathrm{MR})^{25)}$. By contrast, 11ßHSD type 2 inactivates cortisol (Figure-2). In human endometrium, both 11BHSD isoforms as well as GR and MR are expressed. During decidual transformation from endometrial stromal cells, 11ßHSD1, but not type 2 , is extremely induced; this induction is driven sensitively by progesterone $(\mathrm{P} 4)$, leading to the local production of active cortisol ${ }^{26)}$. Predominant cortisol-binding receptors alter GR in undifferentiated cells to MR in decidualising $\mathrm{HESCs}^{26)}$. Implantation is one of the inflammatory responses. The balance of corticosteroid signalling plays a significant role in the control of inflammation and maintenance of early pregnancy in decidualising endometrium.

\section{Regulation of uNK cells via 11ßHSD1/GR signalling in endometrium}

Endometrial receptivity and early pregnancy are controlled by pro- $^{-}$and anti-inflammatory gene networks in decidualising stromal cells underlying the luminal epithelium ${ }^{22)}$. The association between unexplained RPL and elevated uNK cells in midluteal endometrium has been previously reported ${ }^{13)-17)}$. The aberrantly high $\mathrm{uNK}$ cell density plays a role in poor induction of 11BHSD1 and decidual marker genes, such as prolactin (PRL) and insulin-like growth factor-binding protein-1 (IGFBP1), in decidualising HESCs ${ }^{27}$. Degression of 11ßHSD1 expression in decidualising HESCs may lead to insufficient production of cortisol and impaired regulation of GR and MR gene networks at local implantation sites in women with a history of unexplained RPL ${ }^{26)}$. By silencing 11ßHSD1 with carbenoxolone disodium salt or 11ßHSD1 inhibitor, PF915275 attenuated IGFBP1 expression, suggesting impaired decidualisation of HESCs ${ }^{26}$. $11 \beta \mathrm{HSD} 1$ may be a key enzyme as a novel decidual marker in the decidual transformation of endometrium for pregnancy maintenance. 11ßHSD1 has been sufficiently well-conserved during evolution and is also regulated under a hAT-Charlie family DNA transposon (MER20), which contributes to the evolution of mammalian pregnancy, in endometrial stromal cells ${ }^{28}$. On the contrary, glucocorticoid treatment can modulate uNK cell count via GR, which has been demonstrated to be a surface nuclear receptor on uNK cells ${ }^{29}$. Moreover, glucocorticoids also regulate inflammatory cytokines, prostaglandin and $N F-\kappa B$ signalling via GR. In addition, GR in decidualising HESCs is important in epigenetic programming via Krüppel-associated box domain zinc finger proteins (KRAB-ZFPs), which are transcriptional repressors that are associated with heterochromatin formation, thus enhancing the global level of trimethylation of histone $\mathrm{H} 3$ lysine 9 (H3K9me3) ${ }^{26)}$. 11BHSD1/GR signalling is a significant pathway for the regulation of GR network genes, including uNK cells, and inflammatory reaction upon decidualisation of HESCs.

\section{Importance of retinoid metabolism signalling via $11 \beta \mathrm{HSD} 1 / \mathrm{MR}$ signalling in decidualising endometrium}

Endometrial stromal cells with high uNK cell density impaired 11ßHSD1 and MR expressions, but not $\mathrm{GR}^{27)}$. Furthermore, $\mathrm{MR}$ is a predominant cortisol-binding receptor in decidualising HESCs; thus, MR and its gene network must have an essential role in implantation and decidualisation ${ }^{26)}$. DHRS3, an MR-dependent enzyme, was detected from the microarray data of decidualising HESCs following siRNA-mediated MR silencing. DHRS3 in alcohol dehydrogenase and $\operatorname{NADP}(\mathrm{H})$-dependent short-chain dehydrogenases/reductases (SDR) oxidises retinaldehyde (Rald) to retinol and promotes lipid droplet formation ${ }^{30}$. DHRS3 expression was substantially upregulated upon decidualisation, and the appearance of perinuclear lipid droplets in decidualising cells was relatively abundant compared with undifferentiated stromal cells, suggesting that retinoid is stored as retinyl esters in lipid droplets (retinosome) via MR and DHRS3 during decidual transformation of HESCs ${ }^{26}$. In addition, DHRS3 paralleled lipid droplet accumulation in decidualising HESCs with the treatment of MR 


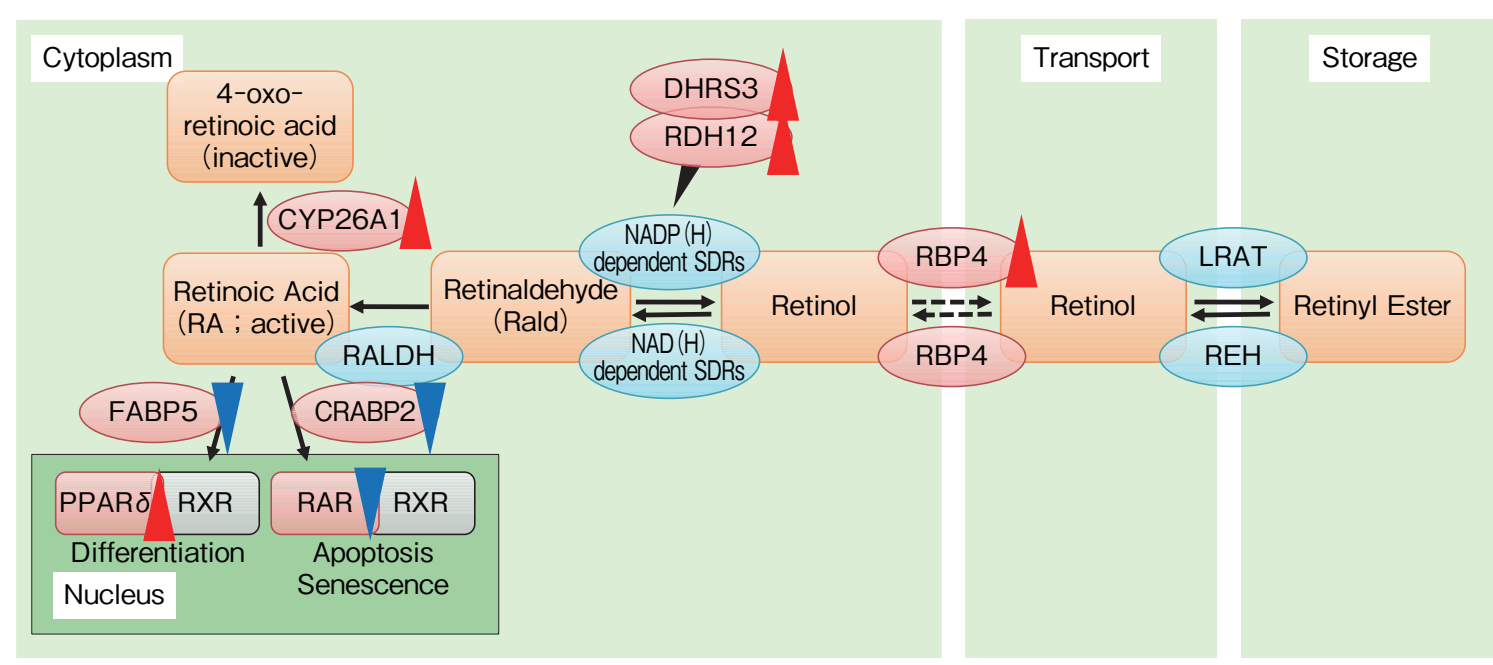

Figure-3 Retinoid metabolism signalling

Retinol (vitamin A), which is hydrolysed from retinyl ester, is transformed to retinaldehyde (Rald) and generates retinoic acid (RA) following two-step oxidation. RA binds to CRABP2 and activates retinoic acid receptor (RAR), leading to apoptosis and senescence. By contrast, RA-dependent activation of PPAR $\beta / \delta$ is mediated through FABP5. Decidualisation of HESCs decreases the expression of CRABP2 and FABP5 and down- and upregulates RAR and PPAR $\beta / \delta$, respectively. In addition, decidualisation is also associated with upregulation of retinol-binding protein 4 (RBP4), cytochrome P450 26A1 (CYP26A1), RDH12 and DHRS3, suggesting that decidualisation suppresses RA signalling by decreasing key cytoplasmic-binding proteins and upregulating retinoid metabolism. Red arrows: gene upregulation upon decidualisation. Blue arrows: gene downregulation.

agonist (aldosterone) and antagonist (RU26752) ${ }^{26)}$. With regard to adipocyte tissue, lipid droplet accumulation completely failed in MR knockout cells $^{31)}$. Moreover, MR downregulation inhibits cell differentiation; thus, MR signalling is significant in MR-dependent adipogenesis ${ }^{32)}$, indicating the link between MR, cell differentiation, and lipid droplet; however, the details are unknown. A study on endometrial cells suggests that retinoid metabolism signalling via MR modulates cell differentiation and lipid droplet accumulation ${ }^{26)}$ 27) 33).

Retinoids are versatile vitamin A (retinol) derivatives that play roles in the regulation of multiple cellular functions and immune tolerance ${ }^{34)}$. In pregnancy, retinoid synthesis is necessary for embryogenesis and foetal development; however, excessive levels are toxic to the embryo and foetus ${ }^{35)}{ }^{36)}$. Retinol, an essential fat-soluble vitamin, is transported to target cells, converted to Rald, and then to all-trans-retinoic acid (RA), which is the most active retinoid metabolite ${ }^{34)}$. ${ }^{37)}$ RA regulates two opposing cell fate decisions depending on the activation of specific pathways: differentiation and apoptosis or senescence. RA bound to cellular RA-binding proteins, CRABP2 and FABP5, activates the retinoic acid receptor (RAR), leading to activation of the apoptotic machinery. By contrast,
RA-dependent activation of peroxisome proliferatoractivated receptor (PPAR) $\beta / \delta$ promotes cellular differentiation ${ }^{38)}$ (Figure-3).

An in vitro endometrial research reported that decidualisation of HESCs decreases the expression of the RA-binding proteins CRABP2 and FABP5 in HESCs and down- and upregulates pro-apoptotic $\mathrm{RAR} \alpha$ and $\mathrm{PPAR} \beta / \delta$, associated with cell differentiation, respectively ${ }^{33)}$. In addition, decidualisation was also related to upregulation of retinol-binding protein 4 (RBP4) and various enzymes involved in the metabolism of RA and its precursor, Rald, such as cytochrome P450 26A1 (CYP26A1), which inactivates RA, and members of the family of SDR, including RDH12 and DHRS3, which are then exported by RBP4 (Figure-3). Treatment of HESCs with RA antagonised the induction of PRL, IGFBP1, and 11ßHSD1 upon decidualisation of HESCs in a dose-dependent manner. Treatment with Rald also attenuated the expression of these decidual genes ${ }^{33}$. The treatment of decidualising cultures with RA or Rald selectively upregulated CRABP2 and RAR expression but not FABP5 or PPAR $\beta / \delta$. Collectively, the data suggest that the decidualisation of HESCs suppresses RA signalling by decreasing key cytoplasmic-binding proteins and by upregulating retinoid metabolism. Yet, 
additional RA exposure is toxic for decidualising HESCs and induces a response that may lead to implantation failure or pregnancy loss ${ }^{33}$.

\section{GR and MR-specific gene networks as a target for treatment of unexplained RPL}

Reprogramming of corticosteroid and retinoid metabolism signalling is important during decidual transformation of HESCs (Figure-4). Moreover, perturbation of decidualisation is associated with pregnancy failure. To treat unexplained RPL, Tang et al. reported a randomised controlled trial using prednisolone treatment for patients with RPL with aberrantly high uNK cell counts. The live birth rate with prednisolone was relatively higher, but there was no significant difference between groups (60\% with prednisolone vs. $40 \%$ with placebo) ${ }^{39}$. Implantation is an inflammatory process via local secretion of pro-inflammatory cytokines and prostaglandins from decidual endometrium ${ }^{40-43)}$. Appropriate prednisolone administration may be difficult for regulation of corticosteroid signalling during implantation. 11ßHSD1 is highly induced in response to $\mathrm{P} 4$ treatment in decidualising HESCs ${ }^{26)}$. Therefore, $\mathrm{P} 4$ treatment can control uNK cells via 11ßHSD1/GR signalling. In addition, regardless of what uNK cells express GR, but not PR, as a surface nuclear receptor, $\mathrm{P} 4$ has an ability to modulate $\mathrm{uNK}$ cells directly via $\mathrm{GR}^{44)}{ }^{45)}$. Remarkably, P4 can bind to MR directly, but this binding upon decidualisation is undetermined ${ }^{46)}$. Notably, P4 supplementation for prevention of unexplained RPL has been reported ${ }^{45)}{ }^{47)}$. Nevertheless, luteal support with P4 activates corticosteroid signalling and GR and MR gene networks, leading to modulation of uNK cells and retinoid metabolism signalling. New trials of targeted therapy at corticosteroid and retinoid metabolism signalling for unexplained RPL are warranted.

\section{Acknowledgments}

I thank Juntendo University, Faculty of Medicine,

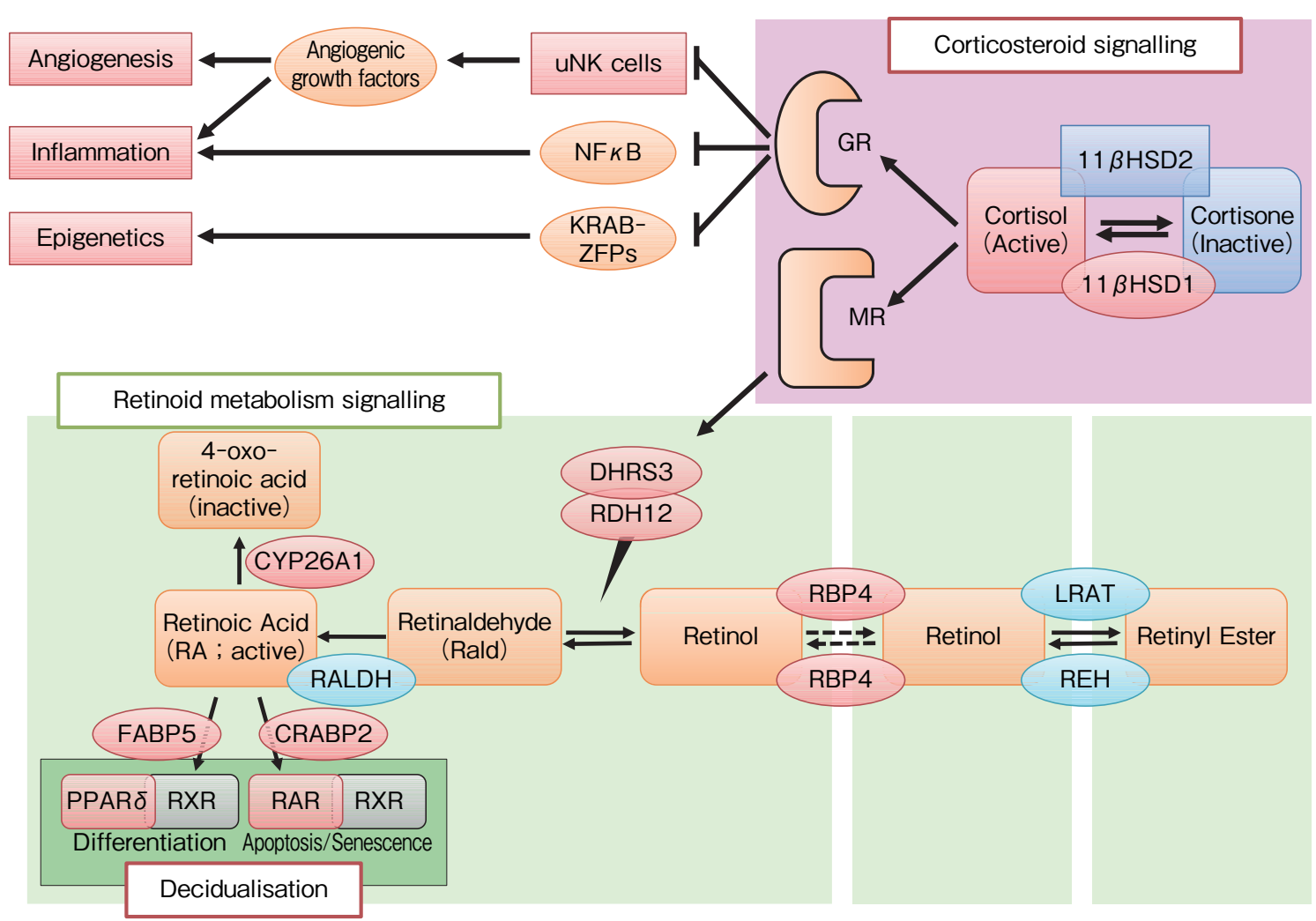

Figure-4 Regulation of decidualisation of endometrial stromal cells via corticosteroid and retinoid metabolism signalling pathways

Corticosteroid signalling via 11ßHSD1 induction regulates GR-specific gene networks including inflammation and angiogenesis at the implantation site. Furthermore, retinoid signalling is controlled via 11ßHSD1/MR signalling, leading to an optimal balance of cell differentiation and apoptosis, such as decidualisation of endometrial stromal cells. 
Graduates' Association for the opportunity to review my research. I also thank Laboratory of Molecular and Biochemical Research, Research Support Center, Juntendo University Graduate School of Medicine, Tokyo, Japan, for the technical assistance. Keiji Kuroda was supported by JSPS KAKENHI Grant Number 18K09273.

\section{Disclosure Statement}

The author has nothing to disclose.

\section{References}

1) Teklenburg G, Salker M, Heijnen C, Macklon NS, Brosens JJ: The molecular basis of recurrent pregnancy loss: impaired natural embryo selection. Mol Hum Reprod, 2010; 16: 886-895.

2) Jacobs PA, Hassold TJ, Henry A, Pettay D, Takaesu N: Trisomy-13 ascertained in a survey of spontaneousabortions. J Med Genet, 1987; 24: 721-724.

3) Ohno M, Maeda T, Matsunobu A: A cytogenetic study of spontaneous-abortions with direct analysis of chorionic villi. Obstet Gynecol, 1991; 77: 394-398.

4) Sugiura-Ogasawara M, Suzuki S, Ozaki Y, Katano K, Suzumori N, Kitaori T: Frequency of recurrent spontaneous abortion and its influence on further marital relationship and illness: The Okazaki Cohort Study in Japan. J Obstet Gynaecol Res, 2013; 39: 126-131.

5) The Eshre Gudeline Group on RPL, Bender Atik R, Christiansen OB, Elson J, et al: ESHRE guideline: recurrent pregnancy loss. Hum Reprod Open, 2018; 2018.

6) Ogasawara M, Aoki K, Okada S, Suzumori K: Embryonic karyotype of abortuses in relation to the number of previous miscarriages. Fertil Steril, 2000; 73: 300-304.

7) Kuroda K: Unexplained recurrent miscarriage: Introduction. In: Kuroda K, Brosens JJ, Quenby S, Takeda S, eds. Treatment Strategy for Unexplained Infertility and Recurrent Miscarriage. Singapore; Springer Singapore; 2018, 79-84.

8) Branch DW, Gibson M, Silver RM: Clinical practice. Recurrent miscarriage. N Engl J Med, 2010; 363: 1740-1747.

9) Teklenburg G, Salker M, Molokhia M, et al: Natural selection of human embryos: Decidualizing endometrial stromal cells serve as sensors of embryo quality upon implantation. PLoS One, 2010; 5: e10258.

10) Weimar CHE, Kavelaars A, Brosens JJ, et al: Endometrial stromal cells of women with recurrent miscarriage fail to discriminate between high- and low-quality human embryos. PLoS One, 2012; 7: e41424.

11) Brosens JJ, Salker MS, Teklenburg G, et al: Uterine selection of human embryos at implantation. Sci Rep, 2014; 4: 3894.

12) Macklon NS, Brosens JJ: The human endometrium as a sensor of embryo quality. Biol Reprod, 2014; 91: 98.

13) Quenby S, Bates M, Doig T, et al: Pre-implantation endometrial leukocytes in women with recurrent miscarriage. Hum Reprod, 1999; 14: 2386-2391.
14) Quenby S, Kalumbi C, Bates M, Farquharson R, Vince G: Prednisolone reduces preconceptual endometrial natural killer cells in women with recurrent miscarriage. Fertil Steril, 2005; 84: 980-984.

15) Quenby S, Nik H, Innes B, et al: Uterine natural killer cells and angiogenesis in recurrent reproductive failure. Hum Reprod, 2009; 24: 45-54.

16) Clifford K, Flanagan AM, Regan L: Endometrial CD56+ natural killer cells in women with recurrent miscarriage: a histomorphometric study. Hum Reprod, 1999; 14: 2727-2730.

17) Tuckerman E, Laird SM, Prakash A, Li TC: Prognostic value of the measurement of uterine natural killer cells in the endometrium of women with recurrent miscarriage. Hum Reprod, 2007; 22: 2208-2213.

18) Manaster I, Mizrahi S, Goldman-Wohl D, et al: Endometrial NK cells are special immature cells that await pregnancy. J Immunol, 2008; 181: 1869-1876.

19) Hanna J, Goldman-Wohl D, Hamani Y, et al: Decidual NK cells regulate key developmental processes at the human fetal-maternal interface. Nat Med, 2006; 12: 1065-1074.

20) Godbole G, Modi D: Regulation of decidualization, interleukin-11 and interleukin-15 by homeobox A 10 in endometrial stromal cells. J Reprod Immunol, 2010; 85: 130-139.

21) Ashkar AA, Black GP, Wei QX, et al: Assessment of requirements for IL-15 and IFN regulatory factors in uterine NK cell differentiation and function during pregnancy. J Immunol, 2003; 171: 2937-2344.

22) Salker MS, Nautiyal J, Steel JH, et al: Disordered IL-33/ST2 activation in decidualizing stromal cells prolongs uterine receptivity in women with recurrent pregnancy loss. PLoS One, 2012; 7: e52252.

23) Huang JY, Su M, Lin SH, Kuo PL: A genetic association study of NLRP2 and NLRP7 genes in idiopathic recurrent miscarriage. Hum Reprod, 2013; 28: 11271134.

24) Lash GE, Bulmer JN, Innes BA, Drury JA, Robson SC, Quenby S: Prednisolone treatment reduces endometrial spiral artery development in women with recurrent miscarriage. Angiogenesis, 2011; 14: 523-532.

25) Arriza JL, Weinberger C, Cerelli G, et al: Cloning of human mineralocorticoid receptor complementary DNA: structural and functional kinship with the glucocorticoid receptor. Science, 1987; 237: 268-275.

26) Kuroda K, Venkatakrishnan R, Salker MS, et al: Induction of $11 \beta-H S D ~ 1$ and activation of distinct mineralocorticoid receptor- and glucocorticoid receptordependent gene networks in decidualizing human endometrial stromal cells. Mol Endocrinol, 2013; 27: 192-202.

27) Kuroda K, Venkatakrishnan R, James S, et al: Elevated periimplantation uterine natural killer cell density in human endometrium is associated with impaired corticosteroid signaling in decidualizing stromal cells. J Clin Endocrinol Metab, 2013; 98: 4429-4437.

28) Lynch VJ, Leclerc RD, May G, Wagner GP: Transposonmediated rewiring of gene regulatory networks contributed to the evolution of pregnancy in mammals. Nat Genet, 2011; 43: 1154-1159.

29) Henderson TA, Saunders PT, Moffett-King A, Groome NP, Critchley HO: Steroid receptor expression in uterine natural killer cells. J Clin Endocrinol Metab, 2003; 88: 440-449. 
30) Deisenroth C, Itahana Y, Tollini L, Jin AW, Zhang YP: p53-inducible DHRS3 is an endoplasmic reticulum protein associated with lipid droplet accumulation. J Biol Chem, 2011; 286: 28343-28356.

31) Hoppmann J, Perwitz N, Meier B, et al: The balance between gluco- and mineralo-corticoid action critically determines inflammatory adipocyte responses. J Endocrinol, 2010; 204: 153-164.

32) Caprio M, Fève B, Claës A, Viengchareun S, Lombès M, Zennaro MC: Pivotal role of the mineralocorticoid receptor in corticosteroid-induced adipogenesis. FASEB J, 2007; 21: 2185-2194.

33) Ozaki R, Kuroda K, Ikemoto Y, et al: Reprogramming of the retinoic acid pathway in decidualizing human endometrial stromal cells. PLoS One, 2017; 12: e0173035.

34) Napoli JL: Retinoic acid biosynthesis and metabolism. FASEB J, 1996; 10: 993-1001.

35) Geelen JA: Hypervitaminosis A induced teratogenesis. CRC Crit Rev Toxicol, 1979; 6: 351-375.

36) Clagett-Dame M, DeLuca HF: The role of vitamin A in mammalian reproduction and embryonic development. Annu Rev Nutr, 2002; 22: 347-381.

37) Kedishvili NY: Enzymology of retinoic acid biosynthesis and degradation. J Lipid Res, 2013; 54: 1744-1760.

38) Schug TT, Berry DC, Shaw NS, Travis SN, Noy N: Opposing effects of retinoic acid on cell growth result from alternate activation of two different nuclear receptors. Cell, 2007; 129: 723-733.

39) Tang AW, Alfirevic Z, Turner MA, Drury JA, Small R, Quenby S: A feasibility trial of screening women with idiopathic recurrent miscarriage for high uterine natural killer cell density and randomizing to prednisolone or placebo when pregnant. Hum Reprod, 2013; 28: 17431752.

40) Chard T: Cytokines in implantation. Hum Reprod Update, 1995; 1: 385-396.

41) Sharkey A: Cytokines and implantation. Rev Reprod, 1998; 3: 52-61.

42) Kelly RW, King AE, Critchley HO: Cytokine control in human endometrium. Reproduction, 2001; 121: 3-19.

43) Bazer FW, Wu G, Spencer TE, Johnson GA, Burghardt RC, Bayless K: Novel pathways for implantation and establishment and maintenance of pregnancy in mammals. Mol Hum Reprod, 2010; 16: 135-152.

44) Guo W, Li P, Zhao G, Fan H, Hu Y, Hou Y: Glucocorticoid receptor mediates the effect of progesterone on uterine natural killer cells. Am J Reprod Immunol, 2012; 67: 463-473.

45) Daya S: Efficacy of progesterone support for pregnancy in women with recurrent miscarriage - a meta-analysis of controlled trials. Br J Obstet Gynaecol, 1989; 96: 275280.

46) Myles K, Funder JW: Progesterone binding to mineralocorticoid receptors: In vitro and in vivo studies. Am J Physiol, 1996; 270: E601-E607.

47) Hussain M, El-Hakim S, Cahill DJ: Progesterone supplementation in women with otherwise unexplained recurrent miscarriages. J Hum Reprod Sci, 2012; 5: 248251. 